\title{
Biodegradation of Polylactide Modified with Starch by Selected Species of Molds
}

\author{
Anna Kowalczyk ${ }^{1,2}$, Emilia Capala ${ }^{1}$, Marek Chyc ${ }^{3}$, Piotr Tyński ${ }^{4}$, Waldemar Sadurski ${ }^{4}$, Tadeusz \\ Kowalski ${ }^{5}$, Dariusz Latowski ${ }^{1}$ \\ ${ }^{1}$ Department of Plant Physiology and Biochemistry, Faculty of Biochemistry, Biophysics and Biotechnology \\ Jagiellonian University \\ Gronostajowa 7, 30-387 Cracow, Poland \\ kowalczyk_anna@onet.eu; emilia.capala@student.uj.edu.pl; dariusz.latowski@uj.edu.pl \\ ${ }^{2}$ Department of Environmental Protection, Faculty of Geology, Geophysics and Environmental Protection \\ AGH University of Science and Technology \\ Mickiewicza 30, 30-059 Cracow, Poland \\ ${ }^{3}$ Department of Environmental Protection, State Higher Vocational School in Tarnów \\ Mickiewicza 8, 33-100 Tarnow, Poland \\ mrsch7@gmail.com \\ ${ }^{4}$ Department of Organic Technologies, New Chemical Syntheses Institute \\ Al. Tysiąclecia Państwa Polskiego 13a, 24-110 Puławy, Poland \\ piotr.tynski@ins.pulawy.pl,waldemar.sadurski@ins.pulawy.pl \\ ${ }^{5}$ Department of Forest Pathology, Mycology and Tree Physiology, University of Agriculture in Cracow \\ Al. 29-Listopada 46, 31-425, Cracow, Poland \\ rltkowal@cyf-kr.edu.pl
}

\begin{abstract}
The new technology of polylactide (PLA) production using starch as an additive compound was developed in order to reduce costs of the process. Two different PLA samples were obtained: with $20 \%$ and $50 \%$ starch concentrations. The comparative analysis of modified and unmodified PLA biodegradation is presented. PLA film with different content of starch was cut to small square-shaped pieces. The samples were disinfected, weighed under sterile conditions and placed on the agar plate with mineral medium. Samples on the agar plate were treated with two species of molds, i.e. Aspergillus niger and Penicillium minioluteum. The cultures were grown for 91 days at $21^{\circ} \mathrm{C}$. Subsequently, the samples were analyzed by weight loss. The modification of foil samples chemical structure was analysed by Attenuated Total Reflectance Fourier Transform Infrared Spectroscopy (ATR-FTIR). To study the effect of microorganisms on structure of the tested PLA samples Scanning Electron Microscope (SEM) was applied.

The greatest weight loss (about 20\%) was observed for the PLA film containing 50\% of starch while the weight loss for the PLA with $20 \%$ of starch was two times lower.

FTIR technique in the analyzed range showed visible changes of PLA spectrum treated with A. niger and P. minioluteum.The changes of signal intensity indicating the reorganization of bonds in tested range of the spectra were observed. The SEM photographs of molds treated samples also demonstrated changes of the PLA film structure which was visibly rough in comparison to smooth surface of the control samples. Chemical and physical changes in the structure of the both species molds treated PLA samples were the clearer, the higher were starch content in PLA.
\end{abstract}

Keywords: polylactide; starch; biodegradation; FTIR; molds

\section{Introduction}

Polylactide (PLA) is a biodegradable aliphatic polyester with thermoplastic properties, which make the polylactide a great material for bioplastic. The properties of this polyester are similar to polyethylene terepththalate (PET) and it can be processed during production by the same methods for thermoplastic substances [1]. PLA is characterized by the possibility of reapplication or hydrolysis to basic compound (lactic acid), what facilitates to broaden its usage for different industrial products [1]. There are two methods of unmodified PLA production [2]. The first method uses lactic acid as a monomer 
and second one uses cyclic form of this acid, lactide (Fig. 1). Producers of PLA add several compounds to improve properties of PLA film, such as corn and potato starch. We tested the biodegradation of PLA modified and unmodified with starch. To verify results of biodegradation FTIR and SEM analyzes were applied. Different forms of polymers degradation, such as chemical, photochemical, thermal or biological result with reorganization, debranching or defragmentation of chain structure, depending on type and conditions of the process. FTIR is universal method widely and successfully used both for biotic and abiotic polymers degradation analyses, as it is very sensible technique for detecting that changes [3-7]. The main factor limiting common usage of PLA is high cost of production and processing of this polymer, therefore the new and cheaper technology of production by starch enrichment. This modification allows to obtain polymer with good technical properties. The main purpose of this work was to investigate capability of modified and unmodified PLA biodegradation.

It is known that in contrast to majority of plastics [8], even unmodified PLA can be subjected to microbial decomposition, therefore by this introduced to biomass life cycle and degraded to $\mathrm{CO} 2$ and water [1], what is significant in terms of environmental protection. The first isolated microorganism capable to biodegrade PLA was soil actinomycetes strain Amycolatopsis HT-32, which degraded 60\% of samples L-PLA with in fourteen days [9]. Another experiment for PLA biodegradation was of Nakamura et al. research [10]. In this study, the bacteria Amycolatopsis sp. strain K104-1 and K104-2 were used. This microorganism degraded over 90\% of emulsified 0.1\% PLA in eight days. Another study on the degradation of PLA was the Li Fan et al. research [11]. In this study Amycolatopsis orientalis degraded PLA in about 80\% in eight days. They isolated the enzymes responsible for the degradation of the PLA from Amycolatopsis genus. The results demonstrated the ability of Amycolatopsis sp. to degrade PLA.

Although there were many experiments conducted with use of bacteria to degrade plastics [12], the molds effect on unmodified and starch modified PLA is still relatively uninvestigated field [13]. In this study the biodegradation by two mold species of unmodified and starch-modified with two different starch concentrations (20\% and 50\%) PLA was tested.

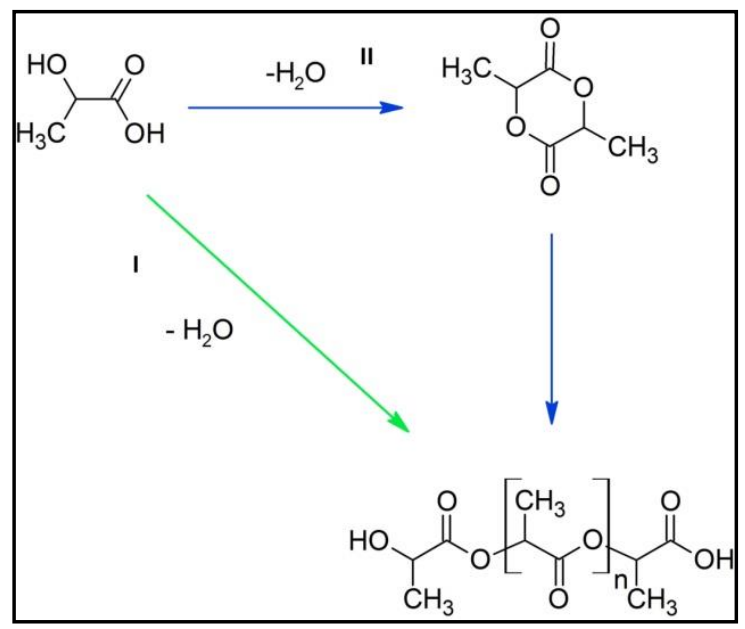

Fig. 1: Different methods of polylactide condensation (green and blue way). The first method (green arrow) has one stage and consists of direct polycondensation of lacitic acid with release of a water molecule. The second method (blue arrows) consists of two steps. The polymerization is preceded by the synthesis of lactide. Lactic acid is subjected to oligomerization with catalysts like zinc and tin salts. Subsequently polymerization is initiated by tin derivatives.

\section{Materials and Methods}

\subsection{Polylactide}

PLA film samples were produced in New Chemical Syntheses Institute (Puławy, Poland). There material was unmodified PLA and modified with starch. The content of starch was $20 \%$ and $50 \%$.

\subsection{Culture Media}

A mineral medium containing $0.1 \mathrm{~g}$ of $\mathrm{NH}_{4} \mathrm{NO}_{3}, 0.05 \mathrm{~g}$ of $\mathrm{KH}_{2} \mathrm{PO}_{4}, 0.05 \mathrm{~g}$ of $\mathrm{MgSO}_{4}$, trace amounts of $\mathrm{FeSO}_{4}$ and $\mathrm{ZnSO}_{4}$ was prepared with $1.5 \%(\mathrm{w} / \mathrm{v})$ agar. 


\subsection{Microorganisms}

Two different species of mold were used. One of them was Aspergillus niger, the most common fungi occurring naturally. This species has got several enzymes making it a great microorganism used in various industries. During previous experiment with A.niger in control sample, which were only PLA film with $20 \%$ of starch in mineral medium a yellowish-green mycelium was observed in the culture. Before the incubation PLA film has been cleaned by rinsing in distilled water, but not disinfected, so that this control sample was infected with different mold. The spores of newly observed mold were isolated and cultivated on agar plate with mineral medium supplemented with sucrose. The organism was identified as Penicillium minioluteum (Fig. 2). This species is very attractively unusual, it produces dextranase, enzyme which hydrolyze $\alpha-1,6$-glycosidic bonds in dextran polymers [14]. The cultivation of both fungi together was favorable for the grow both of them.

\subsection{Degradation of Polylactide by $A$. niger and P. minioluteum in Mineral Medium}

PLA film with different content of starch (20\% and 50\%) and PLA film unmodified was cut to small square-shaped pieces. The samples were disinfected by rinsing in ethanol, weighed under sterile conditions and placed on the agar plate with mineral medium. Samples on the agar plate were treated with two species of mold, i.e. A. niger and P. minioluteum. The cultures were grown for 91 days at $21^{\circ} \mathrm{C}$.

\section{Results and Discussion}

\subsection{Identification of $\boldsymbol{P}$. minioluteum}

The mycelium on MEA agar plates (agar with maltose extract) was yellow with green spores (Fig. 2). Also the medium under the colony was stained slightly red color (Fig. 2C). Those macroscopic features together with microscopic analysis of spores (Fig. 2A) and hyphae (Fig.2B-C) morphologic features match to the description of Penicillium minioluteum [15]. The identification of species was confirmed by genetic analysis. Considering that the PLA film was placed into the medium under sterile conditions, the mold have to come from the PLA film, which was not disinfected. Appearance of $P$. minioluteum imply the fact that PLA film could be a habitat for the mold.

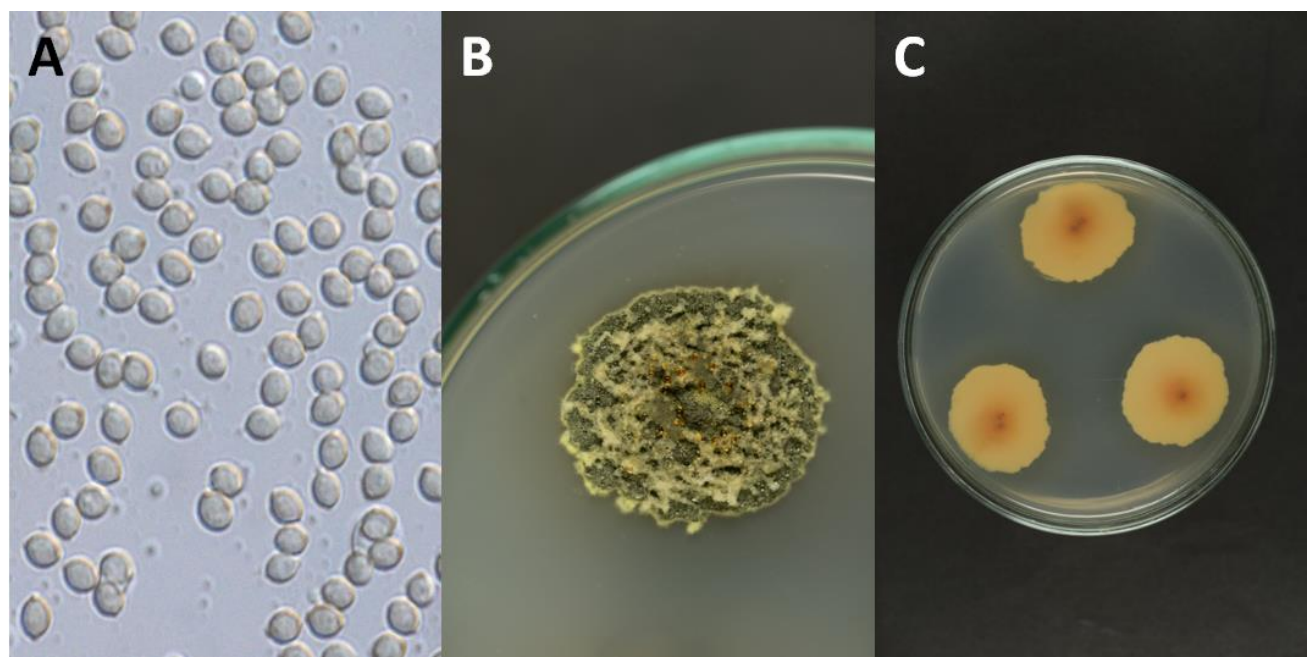

Fig. 2: A-C. P. minioluteum, A - spores, B - colony surface, C - bottom view of the colonies cultured on MEA agar plates.

\subsection{Analysis of Weight Loss}

After the incubation the samples were weighed on an analytical balance and analyzed by weight loss. The greatest weight loss (about 20\%) was observed for the PLA film containing 50\% of starch while the weight loss for the PLA film containing $20 \%$ of starch was about $10 \%$. There was no weight loss detected in case of the unmodified PLA film. 


\subsection{The Chemical Structure}

The modification of foil samples chemical structure was analysed with Attenuated Total Reflectance Fourier Transform Infrared Spectroscopy (ATR-FTIR) with Bruker Alpha-P spectrometer and processed with Opus 6.5 software.

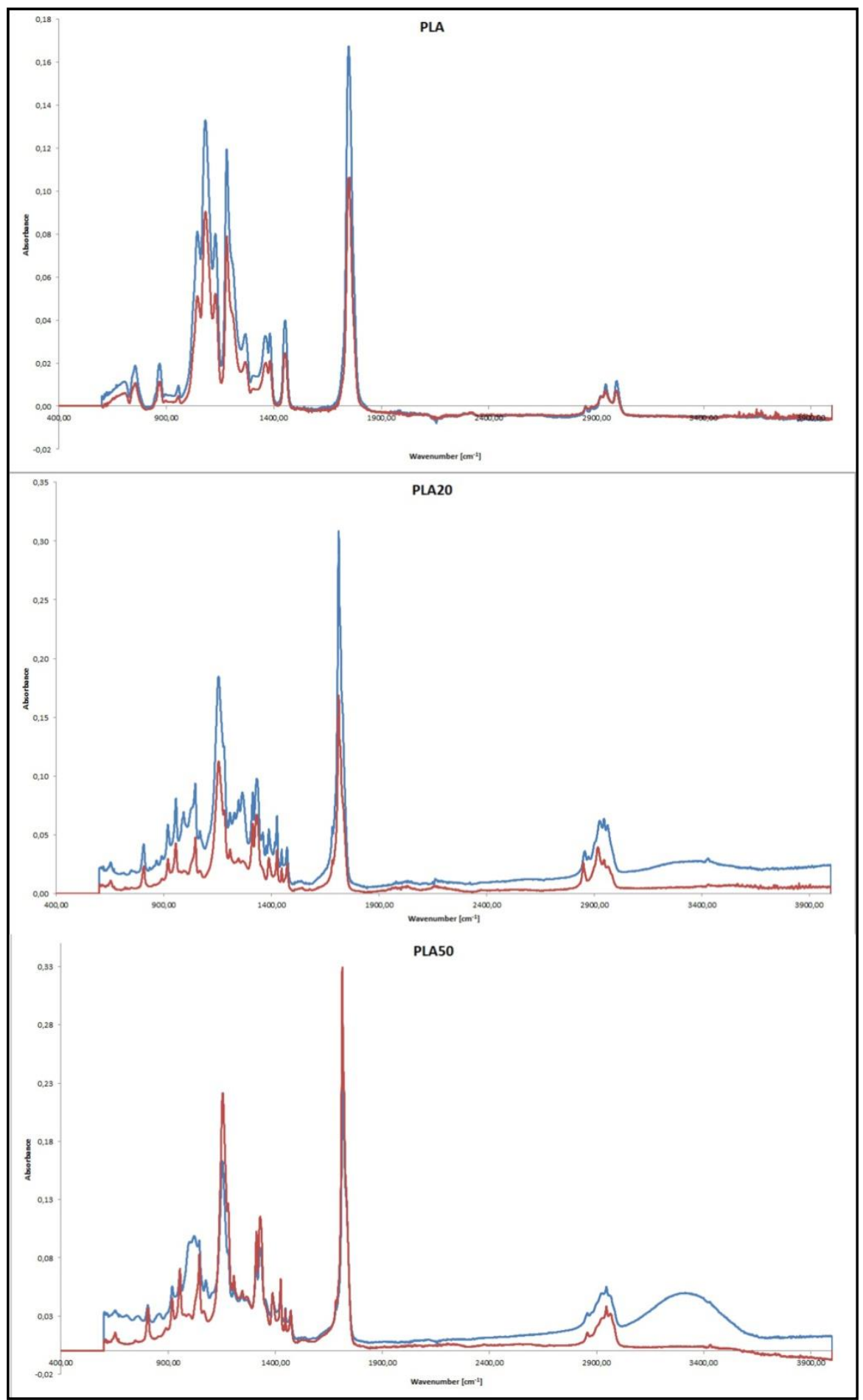

Fig. 3: Comparison of the FTIR spectra. Graphs present the spectrum of control PLA film (blue) and the spectrum of tested PLA film which was incubated with molds (red). The decrease of absorbance is observed for bands at $1100 \mathrm{~cm}^{-1}$ and $1200 \mathrm{~cm}^{-1}, \mathrm{C}-\mathrm{O}-\mathrm{C}$ bonds; $1750 \mathrm{~cm}^{-1}$, dilactones; $2945 \mathrm{~cm}^{-1}$ and $3000 \mathrm{~cm}^{-1}$, -C-H bonds. 
Fourier Transform Infrared Spectroscopy technique in the analyzed range (400-4000 $\mathrm{cm}^{-1}$ ) showed visible changes of PLA spectrum treated with $A$. niger and $P$. minioluteum. The changes of signal intensity indicating modification in polymer chemical structure in tested range of the spectra were observed. The comparison of the control PLA film spectrum and the spectrum of tested PLA film which was incubated with molds is presented in Fig. 3. At 2945 and $3000 \mathrm{~cm}^{-1}$ vibrations originating from $\mathrm{C}-\mathrm{H}$ bands are observed [16]. The bands of this wavenumber from modified PLA are weaker after mold treatment and stronger difference was observed in case of samples with $50 \%$ of starch (Fig. 3). Weakened vibrations at 2945 and $3000 \mathrm{~cm}^{-1}$ may indicate debranching of the PLA chain. Differences observed for vibrations at 1100 and $1200 \mathrm{~cm}^{-1}$ which origin from C-O-C bonds [16], both in case of unmodified PLA and PLA modified with 20\% of starch, when compared to control samples (Fig.3), suggest defragmentation of main PLA chain. The strongest band visible on every spectra at $1750 \mathrm{~cm}^{-1}$ origins from $\mathrm{C}=\mathrm{O}$ aliphatic ester. If it is shifted to approximately $1770 \mathrm{~cm}^{-1}$, indicates dilactone ring, characteristic for lactide monomers [16]. In the case of tested samples the degrading effect of mold activity resulted with weakening of these vibrations (Fig. 3), what may suggest changes in number of $\mathrm{C}=\mathrm{O}$ aliphatic bonds, but the band is not shifted towards bond at $1770 \mathrm{~cm}^{-1}$ specific for monomer structure.

\subsection{The Physical Structure}

To study the effect of microorganisms on the structure of tested PLA samples Scanning Electron Microscope (SEM) was applied. The Scanning Electron Microscope photographs show that the surface of unmodified PLA and PLA with addition of starch is different. The surface of unmodified PLA was smooth in contrast to the surface of PLA with starch which was rough. The differences were the clearer, the higher was starch content in PLA. These differences were also visible in samples treated with molds. On the surface of PLA with 50\% starch content is observed several channels and holes, which indicate that molds were growing into the film. The SEM photographs are presented in Fig. 4.

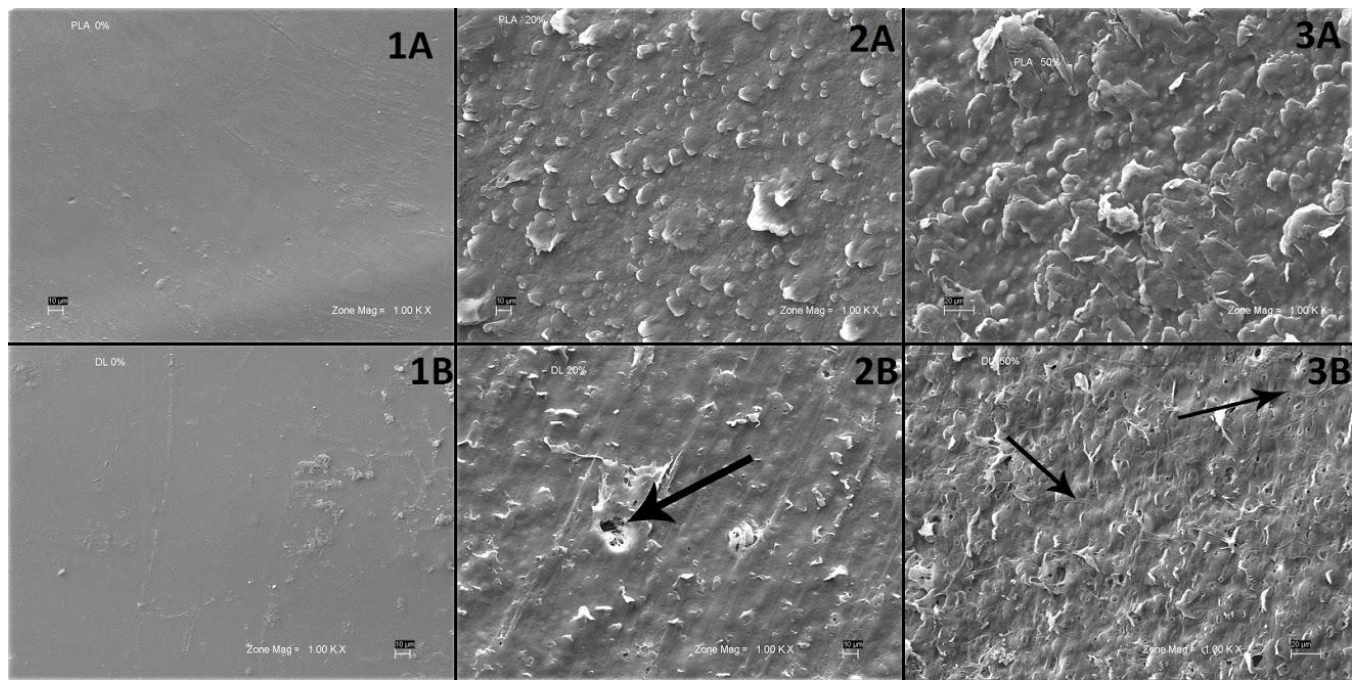

Fig. 4: The Scanning Electron Microscope photographs of PLA film; untreated PLA unmodified (1A) and PLA with addition of starch $20 \%(2 \mathrm{~A})$ and 50\% (3A); PLA treated with molds unmodified (1B) and PLA with addition of starch 20\% (2B) and 50\% (3B). No holes and no channels were observed in the untreated samples, the structure has not been destroyed. There were channels and holes drilled by

molds in the pictures 1B, 2B (arrow) and especially in the picture 3B (arrows) (Fig. 4). There were plenty of visible damage of the structure observed.

\section{Conslusion}

The biodegradation of polylactide modified with starch by selected species of mold was tested. The weight of PLA film decreased after incubation with A. niger and P. minioluteum. In addition FTIR spectra confirmed the damage of PLA structure, indicating changes in chemical structure of PLA chain, particularly with regard to starch content. Chemical and physical changes in the structure of the tested PLA samples were the clearer, the higher were starch content in PLA. To compare the A. niger and $P$. minioluteum capability of PLA biodegradation the experiments on those two species cultivated separately are required. Both studied species are significant for biotechnology - A. niger in citric acid [17] and $P$. 
minioluteum in dextranase production which is used in the sugar-processing industry to decrease viscosity - extensive problem in sugar mills [14]. The optimization of biodegradation process by studied fungi species will enable to use PLA waste as a substrate for industrial production of citric acid and dextranase.

\section{Acknowledgements}

This work was supported by KNOW - The Faculty of Biochemistry, Biophysics and Biotechnology of Jagiellonian University a partner of Leading National Research Center (KNOW) supported by the Minister of Science and Higher Education No. A1/185.

\section{References}

[1] X. Pang, X. Zhuang, Z. Tang, X. Chen, "Polylactic acid (PLA): research, development and industrialization," Biotechnol. J., vol. 5, pp. 1125-1136, 2010.

[2] R. Auras, L. T. Lim, S. Selke, H. Tsuji, "Industrial production of high molecular weight poly (lactic acid)," in Poly(Lactic Acid): Synthesis, Structures, Properties, Processing, and Applications, Ed. New York, John Wiley \& Sons, Inc. Hoboken, 2010.

[3] R. G. Davidson, "Polymer degradation studies by FTIR," in Progress in Pacific Polymer Science 2, Otsu: Springer Berlin Heidelberg, pp. 101-111, 1991.

[4] T. Corrales, F. Catalina, C. Peinado, N.S. Allen, E. Fontanc, "Photooxidative and thermal degradation of polyethylenes: interrelationship by chemiluminescence, thermal gravimetric analysis and FTIR data," J. Photochem. Photobiol. A: Chemistry, vol. 147, pp. 213-224, 2002.

[5] C. Erbetta, G. Manoel, A. Oliveira Rodrigues, M. Ribeiro e Silva, R. Freitas, R. Sousa, "Rheological and thermal behavior of high-density polyethylene (HDPE) at different temperatures," J. Mat. Sci. App., vol. 5, pp. 923-931, 2014.

[6] K. Sanchez, N. Allen, Polyethylene degradation: effect of polymerization catalyst. Society of Plastics Engineers (SPE) Plastics Research, 2011, [Online], Available: http://www.4spepro.org/pdf/003371/003371.pdf

[7] O. M. Immanuel, A. A. Ibiene, H. O. Stanley, "Enhanced biodegradation of polyethylene by fungus isolated from the koluama mangrove swamp in the Niger Delta," J. Microbiol. Biotech. Res., vol. 4, no. 2, pp. 1-9, 2014.

[8] H. Maeda, Y. Yamagata, K. Abe, F. Hasegava, M. Machida, R. Ishioka, K. Gomi, T. Nakajima, "Purification and characterization of biodegradable plastic-degrading enzyme from Aspergilus orizae," Appl. Microbiol. Biotechnol., vol. 67, no. 6, pp. 778-788, 2005.

[9] H. Pranamuda, Y. Tokiwa, H. Tanaka, "Polylactide degradation by an Amycolatopsis sp," Appl. Environ. Microbiol., vol. 63, no. 4, pp.1637-1640, 1997.

[10] K. Nakamura, T. Tomito, N. Abe, Y.Kamio, "Purification and characterization of an extracellular poly (L-lactic acid) depolymerase from a soil isolate, Amycolatopsis sp. strain K104-1," Appl. Environ. Microbiol., vol. 67 no. 1, pp. 345-353, 2001.

[11] F. Li, S. Wang, W. Liu, G. Chen, "Purification and characterization of poly(L-lactic acid)-degrading enzymes from Amycolatopsis orientaliss ssp. Orientalis," FEMS Microbiol. Lett., vol. 282, pp. 52-58, 2008.

[12] R. Premarj, D. Mukesh, "Biodegradation of polymers", Indian J. Biotechnol., vol. 4, pp. 168-193, 2005.

[13] L. Cosgrove, L. McGeechan, G. S. Robson, P. Handley, "Fungal communities associated with degradation of polyester polyurethane in soil," App. Environ. Microbiol., vol. 73, no. 18, pp. 5817-5824, 2007.

[14] A. M. Larsson, R. Andersson, J. Stahlberg, L. Kenne, T. A. Jones "Dextranase from Penicillium minioluteum," Structure, vol. 11 no. 9, pp. 1111-1121, 2013.

[15] T. W. Yenn, A. S. Ngim, D. Ibrahim, L. Zakaria, "Antimicrobial activity of Penicillium minioluteum ED24, an endophytic fungus residing in Orthosiphon stamineus Benth," World World J. Pharm. Sci., vol. 3, no. 3, pp. 121-132, 2014.

[16] L. Nikolic, I. Ristic, B. Adnadjevic, V. Nikolic, J. Jovanovic, M. Stankovic, "Novel microwave-assisted synthesis of poly(D,L-lactide): the influence of monomer/initiator molar ratio on the product properties," Sensors, vol. 10, no. 5, pp. 5063-5073, 2010.

[17] L. P. S. Vandenberghe, C. R. Soccol, A. Pandey, J.-M. Lebeault, "Solid-state fermentation for the synthesis of citric acid by Aspergillus niger," Bioresour. Technol., vol. 74, pp. 175-178, 2000. 